\title{
Die Erzeugung kapitalistischer Realitätsprobleme: Wachstums- regimes und ihre subjektiven Grenzen
}

\author{
Dass die Expansionsdynamik kapitalistischen Wirtschaftens im globalen Maßstab an absolute \\ ökologische Grenzen stößt, wird inzwischen breit problematisiert. Dass die Grenzen des \\ Wachstums im Hinblick auf die Ausweitung des Zugriffs auf menschliche Arbeitskraft bisher \\ weit seltener Beachtung finden, mag auch daran liegen, dass es sich hier weniger um absolute \\ denn um relative und formationsspezifische Wachstumsgrenzen handelt. Um sie bestimmen \\ zu können, ist ein Verständnis unterschiedlicher kapitalistischer Wachstumsregimes als \\ spezifischer Weisen der Erzeugung menschlicher Subjektivität erforderlich. Diese Perspektive \\ soll hier in Eckpunkten umrissen und durch eine Interpretation der kapitalistischen \\ Subjektivierungsgeschichte (West-)Deutschlands als Abfolge dreier solcher Regimes in ihrer \\ Anwendung demonstriert werden.
}

DENNIS EVERSBERG

\section{Einleitung}

Kapitalismus, so könnte man die seit den 1970er Jahren geführten Debatten um die „Grenzen des Wachstums“ zusammenfassen, hat ein Problem mit der Realität: Eine Art und Weise des Wirtschaftens, die auf die potenziell unendliche Ausweitung der Erzeugung von Waren und Dienstleistungen angelegt, dabei aber auf die Verwertung endlicher natürlicher Ressourcen und auf die Absorption schädlicher Nebeneffekte durch ein endliches Ökosystem angewiesen ist, kann auf die Dauer nicht gut gehen. Im Hinblick auf den globalen Verbrauch fossiler Ressourcen und den Klimawandel ist das inzwischen „common sense“ (Randers 2012; Jackson 2011). Kaum gefragt wird indes, ob Vergleichbares nicht auch für den ebenso der Logik dynamischer Steigerung unterliegenden kapitalistischen Zugriff auf die menschliche Arbeitskraft gilt.

Wachstum gibt es nur durch menschliches Tun: „Der Kapitalismus hat die inhärente Fähigkeit, menschliche Energie zu mobilisieren und sie in Wachstum zu transformieren" (Aglietta 2000a, S. 397). Anders als andere natürliche Ressourcen haben Menschen allerdings einen eigenen Willen, der es unmöglich macht, sie in vergleichbarer Weise einfach „aufzubrauchen“. Jede Form, in der die Verwertung menschlicher Arbeitskraft organisiert wird, verlangt bestimmte Fähigkeiten, Haltungen und Vorlieben von den als Arbeitskräfte eingespannten Menschen, und der Zwang zum
Wachstum gebietet, dass diese ihnen in zunehmender Intensität abverlangt werden müssen - bis sie beginnen, sich dagegen zur Wehr zu setzen. Wenn das passiert, erreicht das bisherige Regime seine subjektive Grenze, und es muss etwas Neues an seine Stelle treten, das von den Menschen - zumindest vorübergehend - akzeptiert werden kann. Solange es dabei nicht zu einer grundlegenden Umwälzung kommt, durch die der Wachstumszwang insgesamt außer Kraft gesetzt werden kann, läuft das auf ein neues kapitalistisches Regime der Erzeugung und Vernutzung von Arbeitskraft hinaus. Die subjektiven Grenzen des Wachstums markieren also zugleich eine Triebkraft der bemerkenswerten historischen Anpassungsfähigkeit des Kapitalismus.

Die Untersuchung subjektiver Wachstumsgrenzen setzt voraus, sich nicht an Wachstum als abstraktem Prinzip, sondern an spezifischen, historisch und geografisch eingrenzbaren Wachstumsregimen zu orientieren, diese aber nicht nur als Modelle der Organisation der Produktion von Gütern und Dienstleistungen zu begreifen: Jedes Wachstumsregime erzeugt zugleich einen oder mehrere „Sozialcharaktere" (Lüscher 1990), die zwar von ihm hervorgebracht werden, aber mit den von ihm vorausgesetzten Persönlichkeitstypen nicht identisch sind - und deren Ansprüche, Fähigkeiten und Wünsche deshalb mit den Anforderungen dieses Regimes an die Arbeitskräfte in Konflikt geraten müssen. Als Subjektivierungsweise, so die These, bringt jedes Wachstumsregime also Subjekte hervor, deren innere Verfasstheit über es selbst hinausweist. 
Um zu umreißen, wie sich subjektive Grenzen des Wachstums bestimmen lassen und zu welchen Einsichten ihre Erforschung führen kann, gehe ich in zwei Schritten vor. In Abschnitt 2 wird der regulationstheoretische Begriff des Wachstumsregimes eingeführt und erläutert, wie sich solche Regimes mit Hilfe von Michel Foucaults Kategorie des Dispositivs auch als Subjektivierungsweisen verstehen lassen. In Abschnitt 3 werden diese Begrifflichkeiten angewendet, um an der historischen Abfolge der drei zentralen Wachstumskonstellationen des (west-)deutschen Kapitalismus seit dem 19. Jahrhundert - dem liberalen, dem organisierten und dem flexiblen Kapitalismus - knapp die Möglichkeiten einer vergleichenden Untersuchung von Subjektivierungsregimes und ihrer subjektiven Grenzen zu demonstrieren. Abschnitt 4 zieht ein Fazit und zeigt weitere Forschungsdesiderate auf.

\section{Wachstumsregimes als Subjektivierungsweisen}

Die Verwendung des Begriffs des Wachstumsregimes in der französischen Regulationstheorie basiert auf der Annahme, dass kapitalistische Gesellschaften sich zwar nur durch die stetige Ausweitung wirtschaftlicher Aktivität stabilisieren können, dass diese dynamische Stabilisierung aber weder von selbst noch stets in gleicher Weise eintritt. Vielmehr müssten für dauerhaft stabiles Wachstum eine Reihe gesellschaftlicher Institutionen so zum Zusammenwirken gebracht werden, dass die Ergebnisse ihres Wirkens die stetige Ausweitung eines bestimmten Typs von wirtschaftlichem Output koordiniert befördern. In der regulationstheoretischen Literatur werden dabei in der Regel fünf zentrale ,institutionelle Formen" der Regulation kapitalistischen Wirtschaftens unterschieden: Der „Lohn-Arbeits-Nexus“, das monetäre Regime, die Form des Wettbewerbs, die Einbindung in das internationale Regime sowie die Beziehungen zwischen Staat und Gesellschaft (Aglietta 2000a; Boyer/Saillard 2002). Ein Wachstumsregime komme zustande, wenn diese „Vermittlungsmechanismen" in einer koordinierten Anordnung aufeinander bezogen seien, zu seiner „Erschöpfung“ komme es, wenn sie nicht länger kohärent zusammenwirkten (Aglietta 2000a, S. 405f.). Solche Krisenprozesse sind aber keine Anzeichen für ein Zusammenbrechen des Kapitalismus insgesamt, sondern zeigen gerade seine Erneuerungsfähigkeit an: „Der Regulationsmodus läuft aus dem Ruder und eine Krise bricht aus. Damit beginnt eine Übergangsphase zum Um- und Neubau von Institutionen, bis die neuen innovativen Kräfte der Kapitalakkumulation in einem neuen Wachstumsregime geordnet werden können“" (Aglietta/Bai 2013, S. 4).

\subsection{Dispositive der Steigerung}

Der Gedanke der historischen Abfolge und geografischen Differenzierung von Wachstumsregimes stellt einen hilfreichen theoretischen Rahmen für die Suche nach den subjektiven Grenzen kapitalistischen Wachstums bereit. Auf die Frage, welche Arten von Subjektivität jeder einzelne Wachstumsmodus voraussetzt und erzeugt, hat die Regulationstheorie aus sich heraus jedoch wenig Antworten. Als Ergänzung bietet sich der von Michel Foucault in seinen historischen Studien über Macht und Subjektivierung verwendete Begriff des Dispositivs an. Foucault definiert das Dispositiv erstens als ein „heterogenes Ensemble“ sozial wirksamer Objekte - Diskurse, Praktiken, Technologien, technische Geräte, Formen der Selbstführung usw. (Foucault 2003), denen in ihrer Gesamtheit bestimmte Machtwirkungen ebenso wie Effekte auf die Konstitution der ihnen ausgesetzten Menschen als Subjekte zugeschrieben werden können. Zweitens bezeichnet er damit die Art von „Verkettung“ einer Vielzahl ähnlich strukturierter sozialer (Mikro-)Beziehungen, die mittels jener Diskurse, Sozialtechnologien und Selbstführungsweisen hergestellt wird (Foucault 1977). Indem innerhalb des Dispositivs eine Reihe relativ einheitlicher Kräfte auf die Beziehungen zwischen z. B. Schülerinnen und Lehrerinnen, Zivilistinnen und Uniformierten oder Arbeitskräften und ihren Vorgesetzten einwirken, werden vermittelt durch diese Machtbeziehungen und die an ihnen beteiligten Menschen soziale Realitäten erzeugt. Kurz gesagt, sind Dispositive also zu verstehen als Verhältnisse der Realitätsproduktion. Das beinhaltet die Produktion von Realitäten, die den Subjekten äußerlich sind, zugleich aber auch die der subjektiven Realitäten der Menschen in den ,verketteten“ Machtverhältnissen (Eversberg 2014).

Damit lässt sich ein Wachstumsregime, in Erweiterung des regulationstheoretischen Verständnisses, auch als eine Konstellation von Dispositiven beschreiben, die den Zweck erfüllen, die immanenten Steigerungsimperative des Kapitalismus ins Werk zu setzen: Die unterschiedlichen Dispositive - z. B. Unternehmensformen, Beschäftigungsverhältnisse, Sozialversicherungen, geld- und wirtschaftspolitische Praktiken - sind strategisch so positioniert und aufeinander bezogen, dass ihre Wirkungen die stetige Steigerung des Hervorbringens eines ganz bestimmten Typs von Realität befördern oder erzwingen. Das bezieht sich sowohl auf die Steigerung des Ausstoßes profitabel vermarktbarer Produkte als auch auf die Erzeugung einer sozialen Umgebung, die dem Absatz derselben förderlich ist, sowie von Menschen, die ein Interesse daran haben, sich aktiv an ihrer Erzeugung und ihrem Konsum zu beteiligen. Die Aufmerksamkeit richtet sich damit über die von der Regulationstheorie untersuchten institutionellen Formen hinaus auch auf alles andere, was an der Herstellung produktiver Subjekte und der Beziehungen zwischen ihnen beteiligt ist (Familie, Bildungsinstitutionen, Alltagspraktiken, das Reden und Denken über sich selbst und die Gesellschaft usw.).

So verstanden, bezieht sich der Begriff des Wachstumsregimes auf die Art und Weise, in der kapitalistische Gesellschaften (genauer: Teile von ihnen) als strategisch angeordnete Formationen zur Steigerung bestimmter Formen ökonomischen Outputs begriffen werden können. Es gibt daher nicht eine, sondern eine Vielzahl „kapitalisti- 
scher Produktionsweisen“. Und der Begriff des Wachstumsregimes erlaubt es, wichtige Unterschiede zwischen historischen Phasen der kapitalistischen Entwicklung ebenso wie zwischen unterschiedlichen, zum gleichen historischen Zeitpunkt koexistierenden regionalen Regimes zu benennen.

\subsection{Die Produktion produktiver Subjekte und ihre Grenzen}

Nun ist jede dieser Konstellationen von Dispositiven nicht nur eine Produktionsweise vermarktbarer Güter und Dienstleistungen, sondern zugleich, als notwendige Kehrseite, auch eine Produktionsweise von damit korrespondierenden Subjektivitäten - eine Subjektivierungsweise. Die (wenigen) Forschungen zu Subjektivierungsprozessen, die es bislang gibt, scheinen dabei von der Annahme auszugehen, dass die Art von Subjekten, die ein Wachstumsregime produziert, stets den Arbeitskraftbedarfen der jeweils zu maximierenden Art von Produktion entsprächen, dass also kapitalistische Dispositive in subjektiver Hinsicht stets darauf zielten, sich selbst mit der Subjektivität zu versorgen, die sie brauchen, um den Output weiter zu steigern. So lässt sich etwa mit einem gouvernementalitätsanalytischen Ansatz (vgl. etwa Bröckling 2007; zur Kritik bereits O'Malley et al. 1997) auf der Basis von Programmen (also diskursiven Rationalisierungen) der Machtausübung ohne viel Mühe zeigen, dass die etwa von Ratgeberhandbüchern formulierten oder aus den Begründungen von Gesetzeswerken herauszulesenden „Anrufungen“ der Subjekte in mehrfacher Hinsicht darauf zielen, sie zu motivieren: zur Optimierung ihrer Arbeitskraft für die Verwertung im aktuellen Wachstumsregime, zur Organisation ihres Familienlebens und ihres sonstigen Lebenszusammenhangs in einer Form, die die Optimierung der Arbeitskraft unterstützt, und zu einem Konsumverhalten, das in gleicher Weise der gegenwärtigen ökonomischen Formation angepasst ist. Empirisch ist die Situation allerdings erheblich komplexer, weil die realen Anforderungen, mit denen die Menschen alltäglich konfrontiert sind, sich allenfalls noch mittelbar aus der Rationalität solcher Programmschriften ableiten. Studien, in denen die von solchen „Anrufungen“ Betroffenen direkt gefragt werden, stellen in der Regel fest, dass deren Praktiken mit den Idealbildern der programmatischen Rationalität wenig zu tun haben. Üblicherweise wird das als „eigensinnige Aneignung“ interpretiert (Rau 2010; Truschkat 2008). Hierbei wird aber übersehen, dass die in ihrer Wirkung beobachteten Subjektivierungsmechanismen nicht den programmatischen Rationalisierungen entsprechen, sondern sich stets auf der Mesoebene lokaler institutioneller Umgebungen konstituieren und durch die spezifischen Interessen der Akteure auf dieser Ebene gebrochen sind (Eversberg 2012, 2014).

Daher sind Subjektivierungsweisen nicht als in sich einheitliche Apparate der Herstellung einer gleichförmigen, mit den Interessen „des Kapitals“ konformen subjektiven Realität zu verstehen, sondern eher als Kraftfelder, die einen tenden- ziell vereinheitlichenden, aber im Einzelfall sehr unterschiedlich starken Effekt auf die Subjektivitäten konkreter Menschen ausüben. Weil Unternehmen und Staat, also die ökonomisch wie politisch mächtigsten Akteure, ein gemeinsames Interesse an möglichst großem Wirtschaftswachstum haben, wird der vereinheitlichende Impuls der Dispositive eines Wachstumsregimes sich stets auf die optimale Anpassung der Subjektivitäten der Arbeitenden an die jeweils zu optimierende Art von produktiver Aktivität richten. In gleicher Weise gilt es, die Arbeitsteilung im Haushalt an den Erfordernissen auszurichten, die der Produktionsprozess stellt. Und nicht zuletzt gilt es, die Konsumpräferenzen in Richtung des wachsenden Produktionsausstoßes zu lenken. All das ist in doppelter Hinsicht problematisch, denn einerseits verursachen solche vereinseitigenden Anforderungen früher oder später wachsende Unzufriedenheit sowie physische und psychische Schäden, andererseits verschärft sich in diesen Vereinseitigungsprozessen das Potenzial von Konflikten zwischen Arbeit, sozialen Nahbeziehungen und „konsumtiven“ Aktivitäten. Wenn diese Konflikte auf breiter Front manifest werden und sich in kollektiven Kämpfen der Betroffenen Ausdruck verschaffen, hat das Wachstumsregime seine subjektiven Grenzen erreicht.

\section{Die Metamorphosen des kapitalistischen Subjekts}

Wie sähe nun eine vergleichende Analyse kapitalistischer Wachstumsregimes aus, die diese primär als Subjektivierungsweisen in den Blick nimmt und auf ihre subjektiven Grenzen hin befragt? Skizzieren will ich das an dieser Stelle, indem ich die historische Abfolge dreier idealtypischer Regimes - hier liberaler Kapitalismus, organisierter Kapitalismus und flexibler Kapitalismus genannt - in Westeuropa nachzeichne. Die Unterteilung orientiert sich an der für die regulationstheoretische Theoriebildung zentralen Periodisierung in vorfordistische, fordistische und nachfordistische Phasen (Aglietta 2000b; Boyer 2000). Hiermit kann am deutschen Fallbeispiel einerseits gezeigt werden, durch welche typischen Dispositive jedes dieser Regimes auf die Erzeugung einer bestimmten Art von Subjektivierungseffekten angelegt war; andererseits wird deutlich, wie sich im Zuge der Durchsetzung des Regimes aus den lebensweltlichen Erfahrungsräumen der Menschen heraus gegenläufige, über es hinausweisende Subjektivierungen durchgesetzt haben und wie diese jeweils zur subjektiven Triebkraft der Entstehung eines nachfolgenden Regimes werden konnten.

\subsection{Liberaler Kapitalismus: Kollektivierende Individuierung}

Die zweite Hälfte des 19. Jahrhunderts war in Deutschland die Zeit einer rapiden Industrialisierung. Die Anzahl der 
Industriearbeiterinnen und -arbeiter stieg zwischen 1850 und 1895 von einer Million auf 6 Mio. (Alber 1986, S. 5). Wie in anderen westeuropäischen Ländern handelte es sich bei ihnen mehrheitlich um Menschen, die als „freie“ Lohnarbeiter von der subsistenzwirtschaftlichen Bindung an das Land abgelöst, mental aber noch eng in die von den ländlichen Familienstrukturen geprägten kollektiven Sozialbeziehungen eingebunden waren: Sie waren nichts anderes gewöhnt, als in gegenseitiger Abhängigkeit voneinander zu leben und sich als Kollektiv mit gemeinsam geteiltem Schicksal zu sehen. Der Eintritt in die Lohnarbeit unterwarf sie nun allerdings dem Arbeitsvertrag als individuierendem rechtlichem Dispositiv (Meyer 2010): Der Arbeitsvertrag setzte die in den Fabriken Arbeitenden einer Situation aus, in der sie erstmals als Individuum angesprochen und zugleich gezwungen waren, sich als solches zu verhalten. Dies geschah, indem er sie in Abstraktion von ihren sozialen Bindungen auf die Arbeitsleistung verpflichtete, Zuwiderhandlung mit Bestrafung ihrer Person belegte und sie zugleich vermittels des Lohns als ebenso vereinzeltes Marktsubjekt in die Gesellschaft integrierte.

Innerhalb der Fabriken wurde indes zur Steigerung der Arbeitsproduktivität auf Technologien gesetzt, die die Arbeitenden disziplinierten, zu steter und zügiger Arbeit anhielten und dabei einer beobachtenden Überwachung zugänglich machen sollten. Zu diesen Disziplinardispositiven zählten idealtypisch eine zellenförmige Parzellierung der Fabrik nach dem Muster des Gefängnisses, die organische Anordnung der Arbeitsabläufe als „Manöver“ und ihre kombinatorische Koordination in „Taktiken“ (Foucault 1976, S. 216). Auch bei diesen Dispositiven der Produktion innerbetrieblicher sozialer Realität handelte es sich um individuierende, die Arbeitenden gleichzeitig vereinzelnde und einem einheitlichen Zwang unterwerfende Subjektivierungstechnologien.

Weil in dieser Phase industriell produzierte Güter ohnehin nicht für die gedacht waren, die sie herstellten, und die Nachfrage das Angebot grundsätzlich überstieg, während Arbeitskräfte in großer Zahl verfügbar waren, gab es zunächst aus Sicht der einzelnen Fabrikbesitzer keinen Grund, für den dauerhaften Erhalt von Gesundheit und Leistungsbereitschaft der Arbeiterinnen und Arbeiter zu sorgen: Wachstum war auch ohne ihre Loyalität zu erzielen, sie konnten jederzeit ersetzt werden, und der Lohn erschien nur als Kostenfaktor. Die Folge waren niedrige Löhne und schlechte Arbeitsbedingungen in einer weitgehend rechtlosen Lage - kurz, die Situation der Proletarisierung. Diese aber wurde nicht als Vereinzelung, sondern als kollektive Verelendung erlebt: Außerhalb der betrieblichen Sphäre wurden die noch durch den ländlich-agrargesellschaftlichen Erfahrungsraum geprägten, auf gegenseitiger Abhängigkeit beruhenden Beziehungen des sozialen Nahbereichs zur Widerstandsressource gegen die de-kollektivierende Logik des Arbeitsvertrags. Diese Beziehungen waren der Nährboden, auf dem sich der Lernprozess der Klassenwerdung vollzog, der dann zum Entstehen sozialer, kultureller und politischer Organisationen und schließlich der politischen Arbeiter- bewegung führte (Thompson 1963; Vester 1970). In der zweiten Hälfte der 1860er Jahre war dieser Prozess so weit vorangeschritten, dass nationale Gewerkschaftsorganisationen entstanden, und parallel konstituierte sich die Sozialdemokratie, die bis $1878 \mathrm{zu}$ einem so bedeutenden Machtfaktor geworden war, dass sie durch die „Sozialistengesetze“ für zwölf Jahre verboten wurde.

Bis zum Ende des 19. Jahrhunderts hatten also gerade die individuierenden Dispositive des liberal-kapitalistischen Wachstumsregimes vor dem agrargesellschaftlichen Erfahrungshintergrund der Arbeitskräfte die kollektivierende Bewegung der politischen Formierung der arbeitenden Klasse provoziert - und in deren politischer Macht, mit der sie verstärkt Kompromisse zur Verbesserung der Situation der Arbeitenden erzwang, stieß der liberale Kapitalismus an seine subjektive Grenze (vgl. Lüscher 1990, S. 251).

\subsection{Organisierter Kapitalismus: Individualisierende Kollektivierung}

Der historische Erfolg dieser Bewegung, und zugleich der Preis für die Einbindung der arbeitenden Klasse in die bürgerliche Gesellschaft, war die Etablierung dessen, was Castel das „Sozialeigentum“ nennt: Für den Einzelnen institutionell garantierte Anrechte auf Unterstützungsleistungen der Gesellschaft, die die Nichtverfügung über materielles Eigentum als Absicherung gegen die Risiken des Lebens kompensieren sollten (Castel 2000, 2011; Flora 1986, S. XV). Soziale Sicherheit wurde nun wesentlich durch Dispositive von kollektiv-subjektivierender Stoßrichtung geschaffen. Dazu gehören zunächst die Sozialversicherungen, entstanden unter Bismarck in den 1880er Jahren als eine Art präventives $\mathrm{Zu}$ geständnis an die Macht der (damals verbotenen) Sozialdemokratie. Kranken-, Unfall- und Rentenversicherung beruhten in technischer Hinsicht auf der Entdeckung des Risikos als nur statistisch auf Kollektivebene bestimmbarer und daher auch nur durch kollektive Schutzmechanismen zu bearbeitender, genuin sozialer Kategorie (Ewald 1993). Mit der Reichsversicherungsordnung von 1911 wurde diese Logik auch für Angestellte institutionalisiert und es wurden Witwen- und Waisenrenten eingeführt. Die Versicherungsdispositive (ebd.) erfassten so bereits vor dem Ersten Weltkrieg einen großen Teil der Gesellschaft. Mit der Etablierung der Weimarer Republik wurden weitere Kernelemente der kollektivierenden sozialen Ordnung des organisierten Kapitalismus eingeführt (Alber 1986, S. 8f.): Alte und neue Eliten einigten sich auf den allgemeinen Achtstundentag, öffentliche Arbeitsvermittlung, Tarifautonomie und Schlichtungsregeln für kollektive Arbeitskonflikte; 1920 wurde auch die betriebliche Mitbestimmung gesetzlich geregelt. Nach der Hyperinflation wurde die Sozialhilfe ausgeweitet und auf eine neue rechtliche Basis gestellt, weitere Berufsgruppen wurden von den Sozialversicherungen erfasst, schließlich wurde 1927 die Arbeitslosenversicherung eingeführt.

Die von diesen Dispositiven installierte Realität stellte die von ihnen erfassten Arbeitenden als kollektivierte Sub- 
jekte den Unternehmen und dem Staat gegenüber - als kollektives Konfliktsubjekt wie als anspruchsberechtigte Mitglieder einer versicherten Population. In Deutschland wurde diese Subjektivierungsweise noch zugespitzt durch die Institution des Berufs, ein Dispositiv, dessen Ursprünge in der mittelalterlichen Selbstorganisation des Handwerks liegen. Mittels der Kanonisierung industrieller Arbeit in berufliche Kategorien - als standardisierte Bündel von Fähigkeiten und Fertigkeiten, die die Arbeitskraft in mehrjähriger Ausbildung erwirbt und offiziell zertifiziert bekommt - und der berufsstatusbasierten Struktur der Tarifverträge konnte hier ein vorkapitalistisches Dispositiv für die weitere Befestigung der kollektivierenden Subjektivierung in Dienst genommen werden. Auch in diesem Sinne waren die Rechte der einzelnen Arbeiter Sozialeigentum, insofern sie ihnen nicht als Individuum zustanden, sondern als Mitglied des beruflichen Kollektivs, dem sie angehörten und das diese Rechte stützte und durchsetzte.

„Organisierter Kapitalismus“ (Aglietta 2000a, S. 404; Lash/Urry 1988) bedeutete aber nicht nur kollektive politische Macht der Arbeitenden, sondern auch einen hohen Integrations- und Verflechtungsgrad großer Unternehmen. Und auch aus deren Sicht gab es etwas zu gewinnen durch die soziale Integration der Arbeitenden. In der fordistischen Kopplung von standardisierter Massenproduktion und standardisiertem Massenkonsum nahm die kollektivierende Logik des organisierten Kapitalismus in der Nachkriegsphase für etwa zwei Jahrzehnte die Form eines bemerkenswert dynamischen Wachstumsregimes an. Zentral dafür war, dass die in hoch arbeitsteiliger Betriebsorganisation durch Mechanisierung und Aufgliederung der Arbeitsabläufe erreichten stetigen Produktionszuwächse in Form von Lohnsteigerungen und Sozialleistungen teilweise auch den Arbeitskräften zugute kamen, deren steigende Nachfrage am Binnenmarkt zugleich den Absatz der zusätzlichen Produkte ermöglichte. Der Klassenkompromiss setzte eine für beide Seiten vorteilhafte Aufwärtsspirale in Gang, von der durch steigende Steuer- und Beitragseinnahmen auch der Staat profitierte, der dadurch seinerseits zu einem von weiteren Produktivitätsfortschritten abhängigen „Wachstumsstaat" (Castel 2000, S. 326) wurde.

Der Fordismus war also nicht nur ein Produktionsparadigma, sondern ein ganzes Ensemble von Dispositiven, die zusammen eine soziale Formation in ihrer Gesamtheit erzeugten - nicht nur die Warenproduktion, sondern auch die „Herstellung von Menschen“ war nach einer gemeinsamen Logik organisiert. Das notwendige Gegenstück zur fordistischen Fabrik war dabei die (Klein-)Familie, in der mit dem Prinzip der Liebe eine konträre soziale Logik organisierend sein musste, um die Einseitigkeit der Erfahrungen der Arbeitskräfte in der Produktion abzufedern (Lüscher 1990, S. 234). Daraus resultierte, dass im Rahmen der innerfamiliären Arbeitsteilung Aufgabenprofil und Identität der „Hausfrau und Mutter“ einer ähnlichen Normierung unterlagen wie die beruflich bestimmten Identitätsmuster der meist männlichen Arbeitskräfte.
Aber auch die strukturelle Kollektivierung durch die fordistischen Dispositive setzte sich nicht bruchlos in kollektiv denkende und handelnde Subjekte um, sondern trug erneut die Saat ihres Gegenteils in sich, insofern die so generierten Wohlstandszuwächse die entscheidende Voraussetzung für entstandardisierte Lebensmuster und individualisierte soziale Praktiken außerhalb der formalen ökonomischen und politischen Strukturen waren. Sowohl die Verknüpfung zwischen Produktivität, Lohn, Binnenkonsum und Wachstum wie auch der Wohlfahrtsstaat eröffneten den arbeitenden Subjekten (und den von ihnen Abhängigen) wachsende Räume für die (geld- und warenvermittelte) Gestaltung ihres eigenen Lebenszusammenhangs durch Sport, Urlaub und allerhand andere Freizeitaktivitäten: Praktiken, die nicht direkt von den materiellen Lebensbedingungen der Familie und ihren Notwendigkeiten erfordert wurden (wie zuvor etwa die Eigenarbeit in Haus und Garten), sondern die eine „autonome“, nichtstandardisierte Erzeugung des Selbst und der Sozialbeziehungen ermöglichten. Dadurch wurde die inkorporierte Erfahrung der Kollektivität als Faktor geteilter Wahrnehmungs- und Handlungsschemata nach und nach abgeschwächt. Im Gegenzug griff in der jenseits von Arbeit und Staat gelegenen Sphäre der Lebenswelt die Wahrnehmung zunehmend Raum, eine singuläre, einmalige, irreduzible Person zu sein. Damit schlug die Individuierung der Arbeitenden als „doppelt freie“ Vertrags- und Marktsubjekte unter Bedingungen der Wohlstandsgesellschaft in die Individualisierung um: Die institutionalisierte Kollektivierung durch das Sozialeigentum verschaffte den (nichtsdestoweniger) individuierten Marktsubjekten das Geld und die Zeit, die ihnen den Zugang zu einer wachsenden Palette an Selbstverwirklichungsmöglichkeiten eröffnen, und in diesen Sphären erlebten sie sich zunehmend als einzigartige Individuen mit ganz eigenen Bedürfnissen.

Nun ist die Diagnose der Individualisierung nichts Neues - entscheidend ist aber, dass es die subjektiven Auswirkungen der um sich greifenden „lebensweltlichen“ De-Kollektivierung waren, in denen der Fordismus an seine subjektiven Grenzen stieß: Parallel zu seinen ökonomischen Grundpfeilern begannen Ende der 1960er Jahre auch die „mentalen Infrastrukturen“ (Welzer 2011) des Fordismus zu bröckeln. Manifest wurde nun eine soziale Unruhe, die in Köpfen und Alltag von Menschen in unterschiedlichen Sektoren der fordistischen Gesellschaft seit Langem gegoren hatte. Sie wurzelte in der Erfahrung eines sich vertiefenden Spalts zwischen der Vervielfachung von Möglichkeiten im Privatleben einerseits und der strikten Standardisierung von „Normalbiografien“ (Osterland 1990) in den institutionalisierten Sphären der Gesellschaft andererseits. Je mehr eine autonome Lebensführung außerhalb der standardmäßig zugeschriebenen Rollen von Arbeitnehmerin oder „Hausfrau und Mutter" als reale Möglichkeit erfahrbar wurde, desto größer wurde die Unzufriedenheit mit der Art von Zukunft, die diese Dispositive nicht nur im positiven Sinne sicherten, sondern die sich zugleich auch in ein dauerhaftes 
Schicksal verkehrte: Die Perspektive, ein Leben lang entweder an Kinder und Haushalt oder an eine eintönige Arbeit ohne Verwirklichungspotenziale in untergeordneter Position gefesselt zu bleiben. Gegen diese verordneten Identitäten und berechenbaren monotonen Zukünfte formierte sich nun ein breiter Aufstand von unten, der sich auf mindestens zweifache Weise Ausdruck verschaffte: Individuell und innerhalb der Institutionen versuchten junge Frauen und Männer, den vorgezeichneten Biografien durch Erwerb höherer Bildung zu entfliehen. Kollektiv und antiinstitutionell begannen indes Lehrlinge, Studierende, Frauen und unzufriedene Jugendliche, sich in den schnell anwachsenden neuen sozialen Bewegungen zu organisieren, um ihre Forderungen öffentlich zum Ausdruck zu bringen und mit alternativen Lebensentwürfen zu experimentieren. Gleichzeitig wurde in Serien von wilden Streiks, teils angeführt von migrantischen Arbeitskräften, auch aus den unteren Etagen der fordistischen Arbeitsgesellschaft Unmut laut. Die subjektiven Grenzen des Fordismus waren erreicht: „Nicht moralische Prinzipien oder ein ,stiller ' Wertewandel motivierten die neuen sozialen Bewegungen, sondern die praktische Erfahrung eines nicht legitimierbaren Widerspruchs wachsender und doch verweigerter Freiheitspotenziale“" (Vester et al. 2001, S. 256).

\subsection{Flexibler Kapitalismus - Dividualisierung mit offenem Ausgang}

Dass die organisiert-kapitalistische Gesellschaftsformation passé ist, wird inzwischen kaum noch ernsthaft bestritten. Was allerdings auf sie gefolgt ist, und ob es überhaupt so etwas wie ein einigermaßen kohärentes „Nachfolgemodell“ gibt, ist bis heute Gegenstand erbitterter wissenschaftlicher Debatten (Boyer 2000; Aglietta 2000b; Dörre 2001; Streeck 2009). Hinsichtlich der hier interessierenden Frage nach der Logik von Subjektivierungsweisen wage ich die These, dass sich aus der Vielfalt der entstandardisierten und „desorganisierten“ (Lash/Urry 1988) nachfordistischen Dispositive inzwischen durchaus eine gemeinsame Logik herauslesen und dass sich diese auf den Begriff der Dividualisierung (Eversberg 2014) bringen lässt. Gefördert durch die Folgen der lebensweltlichen Individualisierung und den Geist der antifordistischen Revolten, konfiguriert sich das kapitalistische Wachstumsregime, das wir hierzulande zur Zeit antreffen, um Dispositive herum, in denen die in individualistischer Absicht erkämpfte De-Kollektivierung in die strukturelle „Zerlegung“ der Individuen umschlägt. Dividualisierung hat mindestens drei Dimensionen, in denen sie jeweils durch eine Reihe spezifischer Dispositive ins Werk gesetzt wird. Sie ordnet sich in ein Wachstumsregime ein, in dem die Optimierung des Verhältnisses von Inputs und Outputs durch immer kleinteiligeren Zuschnitt und hoch präzise Allokation der in die Verwertung eingespeisten Ressourcen sowie durch Eliminierung aller Formen von Leerlauf und Verschwendung in produktiven Prozessen geleistet wird.
In der ersten Dimension, der Sachdimension, bedeutet Dividualisierung, dass das für eine solche Produktionsordnung zu schwerfällige und zudem störende Ansprüche begründende Dispositiv „Beruf“ durch das der „Kompetenz“ abgelöst wird: Die Bündelung von Fähigkeiten, Fertigkeiten und Einstellungen zu einem festen, statusbegründenden beruflichen Profil ist aufgeschnürt worden, für zunehmend ausdifferenzierte Tätigkeiten werden tendenziell Arbeitskräfte gesucht, die für einen eng begrenzten Zeitraum ein ganz bestimmtes Set an Kompetenzen zu einem möglichst geringen Preis bereitstellen. Der berufliche „Großhandel“ mit Arbeitskraft geht über in den kompetenzbasierten „Detailhandel“, bei dem es darauf ankommt, in einer instabilen Marktumgebung mit ständig wechselnden Anforderungen stets die richtigen „assets“ im Portfolio zu haben. Ermöglicht wurde das durch die Modularisierung der Ausbildungs- und Studiengänge, durch Anforderungen und Angebote des „lebenslangen Lernens“ sowie durch „neosoziale“ (Lessenich 2008) Politiken der „Aktivierung“ und Responsibilisierung, durch die die Einzelnen auf die aktive Weiterentwicklung und Positionierung ihrer Arbeitskraft am Markt verpflichtet werden sollen. Die Avantgarde dieser kompetenziellen Dividualisierung lässt sich in Fraktionen der kulturellen und künstlerischen Eliten finden. Sie traten schon in den 1960er Jahren als Pioniere von Lebens- und Arbeitsmodellen auf, die den von der antifordistischen Subjektivierungskritik artikulierten Forderungen nach Autonomie und Pluralisierung von Erfahrungen entsprachen. Auf ihren spezifischen Arbeitsmärkten, wo spezielle Qualifikationen und Referenzen schon zuvor wichtiger waren als Berufsabschlüsse, wendeten sie dies bei steigender Arbeitslosigkeit in neuartige, portfolioartige Strategien zur Vermarktung der eigenen Arbeitskraft im Wettbewerb. Diese Strategien sind inzwischen in breitere Arbeitsmarktsegmente durchgesickert und haben sich in der Konkurrenz um knappe und zunehmend unsichere Stellen vom selbst gewählten Lebensmodell zum äußeren, durch betriebliche Personalund öffentliche Arbeitsmarktpolitiken durchgesetzten Zwang transformiert.

Die zweite Dimension der Dividualisierung ist die raumzeitliche: Durch flexibilisierte, auf Projektförmigkeit und internem Wettbewerb beruhende Formen der Unternehmensorganisation und entstetigende, sogenannte atypische Beschäftigungsverhältnisse lockerte sich die Bindung zwischen Betrieb und Arbeitskraft, während Mikroelektronik, Internet und Mobilfunk eine neue Qualität der kurzfristig bedarfsorientierten praktischen Organisation und Koordination der Arbeitsabläufe möglich machten. Damit können die Arbeitskräfte nun auch in Raum und Zeit zerlegt werden: „Wenn wir uns in die Sphäre der Info-Arbeit bewegen, ist es nicht länger notwendig, eine Person auf unbegrenzte Zeit für acht Stunden am Tag gekauft zu haben. Das Kapital rekrutiert keine Menschen mehr, sondern kauft Zeitpakete, getrennt von ihren austauschbaren und zufälligen Trägern.” (Berardi 2009, S. 32). So wird es (sozial)technisch möglich, Arbeitskraft in zeitlicher wie inhaltlicher Hinsicht in 
sub-individuellen Einheiten einzukaufen. Basiseinheit der Integration in die Arbeitsgesellschaft ist damit nicht mehr das Individuum, sondern es können auch einzelne Bestandteile der Person bedarfsangepasst, an wechselnden Orten und für maßgeschneiderte Zeiträume vertraglich gebunden und eingesetzt werden - von der diskontinuierlichen Verpflichtung für kurze Zeiträume in der Leiharbeit bis zur wettbewerblich ausgeschriebenen Vergabe fragmentierter, eng umrissener Programmieraufgaben an IT-Fachleute mit hoch spezialisierten Fähigkeiten („cloud working“).

Die dritte, funktionale Dimension der Dividualisierung ist ein Effekt der seit den 1970er Jahren errichteten Dispositive der Finanzialisierung, mit ihrer breiten Streuung des Eigentums an Unternehmen auf Anlagefonds und kapitalisierte Altersvorsorgerücklagen und der kreditgetriebenen weiteren Expansion der Gütermärkte zur Kompensation der Stagnation der Reallöhne (Kreditfinanzierung von Eigenheimen, Autos usw.). Diese Reorganisation des ökonomischen Raums erlegt dem nachfordistischen Lohnarbeiter innere Spaltungen auf: In oberflächlich verschiedenen, aber strukturell miteinander verbundenen Sphären tritt er als produktiv Arbeitender, als Konsument, als investiv sparender Rentenanwärter, als Immobilienspekulant und als Steuerzahler auf. Diese Teilidentitäten sind sämtlich auf das ökonomische Feld bezogen und können im finanzialisierten Kapitalismus nicht mehr widerspruchsfrei nebeneinander bestehen, sondern begründen teilweise vollkommen konträre Interessenlagen. Den Arbeitenden selbst ist nun die Aufgabe angetragen, derart fragmentiert zu leben oder aber seine eigenen widerstreitenden Interessen aktiv zu vermitteln, um den eigenen Lebenszusammenhang individuell zu machen.

Über alle drei Dimensionen hinweg markieren diese Veränderungen einen Bruch mit der Kollektivierungslogik des Fordismus, nicht aber mit den kapitalistischen Steigerungszwängen selbst. Vielmehr hat sich der dekollektivierende Impuls der Widerstände gegen den Fordismus auf der Strukturebene durchgesetzt, ist aber seinerseits der Steigerungslogik unterworfen und bis unter die Ebene des Individuums vorangetrieben worden. Anders als zu Zeiten der liberal-kapitalistischen Proletarisierung haben die Einzelnen nun aber nicht ohne weiteres Zugriff auf kollektive Widerstandsressourcen gegen diese Zerlegung, sodass im Ergebnis das Leben den Imperativen der Arbeit unterworfen und die Verantwortung für die eigene Individualität den Einzelnen übertragen werden kann. Die Dispositive, mittels derer das bewerkstelligt wird, gehören der Ordnung der Kontrolldispositive (Deleuze 1993) an: Die Arbeitsleistung der Subjekte wird im Idealfall nicht durch ihre vertragliche Verpflichtung sichergestellt, sondern dadurch, dass sich ihre Arbeitsprozesse in den digitalen Welten der Computernetze vollständig abbilden. Ihr Anspruch auf Entlohnung ergibt sich der zugrunde liegenden Logik zufolge direkt aus den ständig erfassten, permanent kontrollierbaren Parametern ihres Outputs, und nur wenn sich dieser so in die koordinierten Leistungen der anderen verstreuten und nach Bedarf in die Produktion eingespannten Arbeitskräfte einfügt, dass die Gesamtproduktivität stetig steigt, ist ihnen ein dauerhaftes Auskommen garantiert.

\section{Was hier offen bleiben muss}

Um die Anwendbarkeit und Nützlichkeit der in Abschnitt 2 entwickelten Kategorien zur Analyse kapitalistischer Wachstumsregimes als Subjektivierungsweisen zu demonstrieren, habe ich in Abschnitt 3 in groben Zügen die Konturen der Abfolge von Wachstums- und Subjektivierungsregimes in Westeuropa von der liberalen über die organisierte bis zur flexiblen kapitalistischen Formation umrissen. Der individuierende liberale Kapitalismus, so habe ich dabei zu zeigen versucht, produzierte kollektiv sozialisierte und kampfbereite Proletarierinnen und Proletarier, die den Übergang zum organisierten Kapitalismus (mit) erzwangen. Und Letzterer erzeugte individualisierte Konsumentinnen und Konsumenten, an deren Vorlieben und Praktiken die nachfordistische Flexibilisierung andocken konnte. Die zuletzt gelieferte Beschreibung des flexibel-kapitalistischen Regimes muss allerdings einstweilen unvollständig bleiben, denn es lässt sich auf dem gegenwärtigen Stand von Geschichte wie Forschung noch nicht mit Sicherheit sagen, welche Formen von über ihn selbst hinausweisender „lebensweltlicher“ Subjektivierung der dividualisierende flexible Kapitalismus hervorbringen wird.

Spekulation ist freilich immer möglich: So legen breite Debatten über steigende Fallzahlen von Burnout, Depression und anderen erschöpfungsbedingten Krankheitsphänomenen, steigenden Psychopharmakakonsum und verwandte Phänomene nahe, zu vermuten, dass die extrem hohen kognitiven und sozialen Anforderungen des zeitgenössischen Regimes bereits jetzt an handfeste Grenzen des seelisch und körperlich Verkraftbaren stoßen (vgl. Neckel/ Wagner 2013 und diess. in diesem Heft). Das würde die Fragen aufwerfen, ob und in welcher Weise diese Formen des Leidens zu einem politischen Faktor werden können, der Veränderung erzwingt, und ob es sich dabei dann um den Übergang zu einem neuen, erneut entflexibilisierten und ein anderes Verhältnis zwischen Individuum und Gesellschaft erzeugenden Wachstumsregime handelte, oder ob nun doch der Kapitalismus selbst infrage stünde, weil sich die Möglichkeiten weiterer Effektivierung der Ausbeutung des Menschen angesichts massenhafter psycho-physischer Erschöpfung selbst „kategorial erschöpft“ (Offe 1972, S. 24) hätten, er also mithin doch eine absolute Grenze erreicht habe. Gegenwärtig lassen sich einige Anzeichen so deuten, dass der gemeinsame Nenner von anti-dividualistischen Subjektivierungsformen in einer Sehnsucht oder Suche nach Gemeinschaft - sei es durch den Rückzug in Kleinfamilie und Eigenheim, durch den Konsum idealisierter Darstellungen des „einfachen Landlebens“ oder durch die identitäre Separierung in subkulturellen Szenen - liegen könnte. Allein: Ob daraus ein politischer Faktor werden könnte, 
muss fraglich erscheinen, denn hinter die seit dem liberalen Kapitalismus durchgesetzte Individuierung als Marktsubjekt gibt es unter kapitalistischen Verhältnissen kein Zurück.

Festzuhalten sind abschließend eine Schlussfolgerung und ein gewaltiges Desiderat. Die Schlussfolgerung aus dem Schnelldurchgang durch die kapitalistische Subjektivierungsgeschichte setzt an einer Einschätzung an, die Lüscher (1990, S. 234f.) zugespitzt über den Fordismus formuliert: „,Mit dem Sozialcharakter, den der Fordismus produziert, kann er sich nicht reproduzieren, sondern einzig mit dem, den er auflöst. " Diese Feststellung lässt sich hypothetisch auf jedes kapitalistische Wachstumsregime übertragen: Insofern ein solches immer auch eine oder mehrere Subjektivierungsweise(n) umfasst, lässt sein „ordnungsgemäßes“ Funktionieren im Alltagsleben jenseits von Staat und Betrieb immer Subjektivitäten entstehen, die sich von den durch seine Dispositive vorausgesetzten unterscheiden und sukzessive mit diesen in Konflikt geraten. Das „Realitätsproblem“ des Kapitalismus besteht in subjektiver Hinsicht also weniger darin, dass das kapitalistische Wirtschaften ein real endliches Ressourcenvolumen irgendwann aufgebraucht hätte, sondern darin, dass die Realitäten, die aus einer bestimmten Konstellation seines Operierens hervorgehen, zwangsläufig auf die innere Aushöhlung ebendieser Konstellation hinauslaufen - und in dieser Hinsicht könnte man sagen, dass es den Kapitalismus nur deshalb noch gibt, weil er aus sich heraus regelmäßig an seine Grenzen stoßen muss.

Das unübersehbare Desiderat besteht indes darin, dass jegliche ernst zu nehmende Forschung zum Thema erst noch zu beginnen wäre. Es gälte, die Konturen der hier nur holzschnittartig dargestellten Transformationen historischer kapitalistischer Subjektivierungsweisen erheblich zu schärfen, die diversen auf sie bezogenen Dispositive vollständiger zu benennen, ihre strategischen Funktionen und Wirkweisen detaillierter darzustellen und systematischer auf die Rolle sozialer Akteure im Entstehen und Vergehen von Dispositiven einzugehen. Vor allem aber wäre es ein ungeheuer wichtiges Unterfangen, den Fokus einer solchen Analytik hin zu einer komparativen Perspektive zu weiten und auch andere, insbesondere außereuropäische, Wachstumsregimes auf die mit ihnen korrespondierenden Subjektivierungsweisen und subjektiven Grenzen zu befragen.

\section{LITERATUR}

Aglietta, M. (2000a): A theory of capitalist regulation. The US experience, London et al.

Aglietta, M. (2000b): Ein neues Akkumulationsregime. Regulationstheorie auf dem Prüfstand, Hamburg

Aglietta, M./Bai, G. (2013): China's development. Capitalism and empire, London et al.

Alber, J. (1986): Germany, in: Flora, P. (Hrsg.): Growth to limits. The Western European welfare states since World War II, Vol. 2: Germany, United Kingdom, Ireland, Italy, Berlin/New York, S. 1-154

Berardi, F. (2009): Precarious rhapsody. Semiocapitalism and the pathologies of the post-alpha generation, London

Boyer, R. (2000): Is a finance-led growth regime a viable alternative to fordism? A preliminary analysis, in: Economy and Society 29 (1), S. 111-145 Boyer, R./Saillard, Y. (Hrsg.) (2002): Régulation theory. The state of the art, London/New York

Bröckling, U. (2007): Das unternehmerische Selbst. Soziologie einer Subjektivierungsform, Frankfurt a. M.

Castel, R. (2000): Die Metamorphosen der sozialen Frage, Konstanz Castel, R. (2011): Die Krise der Arbeit. Neue Unsicherheiten und die Zukunft des Individuums, Hamburg

Deleuze, G. (1993): Postskriptum über die Kontrollgesellschaften, in: Ders. Unterhandlungen. 1972-1990, Frankfurt a. M., S. 254-262
Dörre, K. (2001): Gibt es ein nachfordistisches Produktionsmodell? Managementprinzipien, Firmenorganisation und Arbeitsbeziehungen im flexiblen Kapitalismus, in: Candeias, M./Deppe, F. (Hrsg.): Ein neuer Kapitalismus?, Hamburg, S. 83-107 Eversberg, D. (2012): Dispositive und Dispositionen der Arbeitskraft. Aktivierende Arbeitsmarktpolitik als Praxis der Erzeugung von Feldakteuren, in: Bernhard, S./Schmidt-Wellenburg, C. (Hrsg.): Feldanalyse als Forschungsprogramm, Bd. 2, Wiesbaden, S. 109-136

Eversberg, D. (2014): Dividuell aktiviert. Wie Arbeitsmarktpolitik Subjektivitäten produziert, Frankfurt a. M./New York

Ewald, F. (1993): Der Vorsorgestaat, Frankfurt a. M.

Flora, P. (Hrsg.) (1986): Growth to limits. The Western European welfare states since World War II, Vol. 2: Germany, United Kingdom, Ireland, Italy, Berlin/New York

Foucault, M. (1976): Überwachen und Strafen. Die Geburt des Gefängnisses, Frankfurt a. M.

Foucault, M. (1977): Sexualität und Wahrheit. Erster Band: Der Wille zum Wissen, Frankfurt a. M.

Foucault, M. (2003): Das Spiel des Michel Foucault, in: Ders.: Dits et ecrits. Schriften in vier Bänden, Bd. 3, Frankfurt a. M., S. 391-429

Jackson, T. (2011): Wohlstand ohne Wachstum. Leben und Wirtschaften in einer endlichen Welt, München

Lash, S./Urry, J. (1988): The End of organized capitalism, Madison

Lessenich, S. (2008): Die Neuerfindung des Sozialen. Der Sozialstaat im flexiblen Kapitalismus, Bielefeld

Lüscher, R. M. (1990): Henry und die Krümelmonster. Versuch über den fordistischen Sozialcharakter, Tübingen

Meyer, L. (2010): Das Recht des Kapitalismus. Überlegungen zu den monetären Implikationen der Entstehung des modernen Rechts, in: Pahl, H./Meyer, L. (Hrsg.): Gesellschaftstheorie der Geldwirtschaft. Soziologische Beiträge, Marburg, S. $127-164$

Neckel, S./Wagner, G. (Hrsg.) (2013): Leistung und Erschöpfung. Burnout in der Wettbewerbsgesellschaft, Berlin

Offe, C. (1972): Strukturprobleme des kapitalistischen Staates. Aufsätze zur Politischen Soziologie, Frankfurt a. M.

O'Malley, P./Weir, L./Shearing, C. (1997): Governmentality, criticism, politics, in: Economy and Society 26 (4), S. 501-517

Osterland, M. (1990): „Normalbiographie“ und "Normalarbeitsverhältnis", in: Hradil, S./Berger, P. (Hrsg.): Lebenslagen, Lebensläufe, Lebensstile, Soziale Welt, Sonderband 7, S. 352-362

Randers, J. (2012): 2052: Der neue Bericht an den Club of Rome. Eine globale Prognose für die nächsten 40 Jahre, München

Rau, A. (2010): Psychopolitik. Macht, Subjekt und Arbeit in der neoliberalen Gesellschaft, Frankfurt a. M./New York

Streeck, W. (2009): Re-forming capitalism. Institutional change in the German political economy, Oxford/New York

Thompson, E. P. (1963): The making of the English working class, London Truschkat, I. (2008): Kompetenzdiskurs und Bewerbungsgespräche:

Eine Dispositivanalyse (neuer) Rationalitäten sozialer Differenzierung, Wiesbaden Vester, M. (1970): Die Entstehung des Proletariats als Lernprozeß, Frankfurt a. M. Vester, M./von Oertzen, P./Geiling, H./Herrmann, T./Müller, D. (2001): Soziale Milieus im gesellschaftlichen Strukturwandel. Zwischen Integration und Ausgrenzung, Frankfurt a. M.

Welzer, H. (2011): Mentale Infrastrukturen. Wie das Wachstum in die Welt und in die Seelen kam, Berlin

\section{AUTOR}

DENNIS EVERSBERG, Dr., ist Wissenschaftlicher Mitarbeiter am DFG-Forschungskolleg „Postwachstumsgesellschaften“ der Friedrich-Schiller-Universität Jena. Arbeitsschwerpunkte: Macht- und Subjektivitätsanalyse, Arbeits- und Arbeitsmarktsoziologie, Kapitalismusforschung

dennis.eversberg@uni-jena.de 